\title{
193. On Closed Graph Theorem
}

\author{
By Michiko NaKamura \\ (Comm. by Kinjirô Kunugi, M. J. A., Oct. 12, 1970)
}

This paper is to give a type of closed graph theorem for topological linear spaces similar to the one discussed in the previous paper [4], generalizing and simplifying the results obtained in [1], [2], and [3].

We make use of the notations in [4].

A filter $\Phi$ in a linear space $E$ is said to be a LS-filter if $\Phi$ is generated by the complements of all the finite union of linear subspaces $E_{n}(n=1,2, \cdots)$ such that $E=\bigcup_{n=1}^{\infty} E_{n}$.

A subset $A$ of a linear space $E$ is said to be linearly open if for any straight line $L$ in $E, L \cap A$ is open in $L$ by its usual topology.

A filter $\Phi$ in a linear space $E$ is said to be a $P$-filter if for every $x$ in $E$ there exists a linearly open set $A$ such that either $A$ is disjoint from $\Phi$ or $\Phi_{A}$, considered as a filter in $E$, is finer than a $L S$-filter. (In general, we identify a filter $\Psi$ in a subset of $E$ with a filter in $E$ generated by $\Psi$.)

A linear topological space $E$ (in the sequel we suppose that every linear topological space is Hausdorff) is called a generalized netted space (called $G N$-space in the sequel) if there exists a sequence of $P$ filters $\Phi_{n}(n=1,2, \ldots)$ such that every ultrafilter $\Psi$ with $\Psi \nsupseteq \Phi_{n}(n=1$, $2, \ldots)$ converges in $E$.

$E$ is called a pre-GN-space if there exists a sequence of $P$-filters $\Phi_{n}(n=1,2, \cdots)$ such that every ultrafilter $\Psi$ with $\Psi \nsupseteq \Phi_{n}(n=1,2, \cdots)$ is a Cauchy-filter in $E$. The $P$-filters $\Phi_{n}$, in these cases, are called defining filters for $E$.

Let $\varphi$ be a linear mapping from a linear space $E$ into a linear space $F$. The image $\varphi(A)$ of a linearly open subset $A$ of $E$ by $\varphi$ is linearly open in $\varphi(E)$ and the inverse image $\varphi^{-1}(B)$ of a linearly open subset $B$ in $\varphi(E)$ by $\varphi$ is linearly open in $E$.

If $\varphi$ is an one-to-one linear mapping from $E$ into $F$, then the image $\varphi(\Phi)$ of a $P$-filter $\Phi$ in $E$ is a $P$-filter.

If $\varphi$ is a linear mapping from $E$ into $F$, then the inverse image $\varphi^{-1}(\Phi)$ of a $P$-filter $\Phi$ in $F$ such that $\varphi(E)$ is not disjoint from $\Phi$ is a $P$ filter in $E$. In particular, if $E$ is a linear subspace of $F$, then for every $P$-filter $\Phi$ in $F$ such that $E$ is not disjoint from $\Phi, \Phi_{E}$ is a $P$-filter in $E$, and a $P$-filter in $E$ can be considered as a $P$-filter in $F$.

By virtue of these facts, we can see easily that the class of $G N$ - 
spaces, as in the case of quasi-Souslin spaces (in [4]), is closed by the following operations :

(1) The image $E=\varphi(F)$ of a GN-space $F$ by a continuous linear mapping $\varphi$ is a GN-space.

(2) The closed subspace $E$ of a GN-space $F$ is a GN-space.

(3) The product space $E=\prod_{n} E_{n}$ of $G N$-spaces $E_{n}(n=1,2, \cdots)$ is a GN-space.

(4) The inductive limit $E$ of $G N$-spaces $E_{n}(n=1,2, \ldots)$ is a $G N$ space.

First we prove that every metric linear space $E$ is a pre- $G N$-space. Let $d$ be the distance function in $E$. Put $U_{n}(x)=\left\{y \in E \mid d(x, y)<\frac{1}{n}\right\}$. Let $\Phi_{n}$ be the filter generated by the complements of all the finite union of $U_{n}(x)$ for all $x$ in $E$. Then $\Phi_{n}$ is obviously a $P$-filter in $E$ and we show that $E$ is a pre- $G N$-space with the defining filters $\Phi_{n}(n=1,2, \ldots)$. Let $\Psi$ be an ultrafilter in $E$ such that $\Psi \triangleright \Phi_{n}$ for every $n$, then there exists a sequence of elements $x_{n}(n=1,2, \ldots)$ in $E$ such that $\Psi \ni U_{n}\left(x_{n}\right)$. Then $\Psi$ is a Cauchy-filter in $E$.

Proposition. Let $E$ be a linear topological space with a system of subspaces $E_{n_{1}, n_{2}, \cdots, n_{k}}$ defined for every finite sequence $n_{1}, n_{2}, \cdots, n_{k}$ of natural numbers such that

$$
\begin{aligned}
E & =\bigcup_{n=1}^{\infty} E_{n}, E_{n_{1}}=\bigcup_{n=1}^{\infty} E_{n_{1}, n}, \cdots, \\
E_{n_{1}, n_{2}, \cdots, n_{k}} & =\bigcup_{n=1}^{\infty} E_{n_{1}, n_{2}, \cdots, n_{k}, n}, \cdots .
\end{aligned}
$$

Suppose a topology of pre-GN-space is given on each $E_{n_{1}, n_{2}, \cdots, n_{k}}$ such that for every infinite sequence $\left\{n_{k}\right\}_{k=1,2, \ldots}$, if the restriction of a filter $\Psi$ in $E_{n_{1}, n_{2}, \cdots, n_{k}}$ is a Cauchy-filter for every $k=1,2, \cdots$, then $\Psi$ converges in $E$. Then $E$ is a GN-space.

Proof. For each $\left\{n_{1}, n_{2}, \cdots n_{k}\right\}$, let $\Phi_{n_{1}, n_{2}, \ldots, n_{k}}^{i}(i=1,2, \ldots)$ be defining $P$-filters for $E_{n_{1}, n_{2}, \cdots, n_{k}}, \Psi_{n_{1}, n_{2}, \cdots, n_{k}}$ the filter generated by the complements in $E_{n_{1}, n_{2}, \cdots, n_{k}}$ of all the finite union of $E_{n_{1}, n_{2}, \cdots, n_{k}, n}(n=1$, $2, \cdots)$, and $\Psi_{0}$ the filter generated by the complement in $E$ of all the finite union of $E_{n}(n=1,2, \cdots)$. We will prove that $E$ is a $G N$-space with the defining filters $\Phi_{n_{1}, n_{2}, \cdots, n_{k}}^{i}, \Psi_{n_{1}, n_{2}, \cdots, n_{k}}\left(i, n_{1}, n_{2}, \cdots, n_{k}\right)$ and $\Psi_{0}$, these filters being consider as $P$-filters in $E$. Let $\Psi$ be an ultrafilter in $E$ such that $\Psi \nsupseteq \Phi_{n_{1}, n_{2}, \cdots, n_{k}}^{i}, \Psi \nsupseteq \Psi_{n_{1}, n_{2}, \cdots, n_{k}}$ for every $i, n_{1}, n_{2}, \cdots, n_{k}$ and $\Psi \nsupseteq \Psi_{0}$. From $\Psi \nsupseteq \Psi_{0}$ there exists a natural number $n_{1}$ such that $\Psi \ni E_{n_{1}}$ and from $\Psi \nsupseteq \Psi_{n_{1}}$ there exists a nutural number $n_{2}$ such that $\Psi \ni E_{n_{1}, n_{2}}$. Continuing this process, we can find a sequence $\left\{n_{k}\right\}_{k=1,2} \ldots$ such that every $E_{n_{1}, n_{2}, \cdots, n_{k}}(k=1,2, \cdots)$ belongs to $\Psi$. Since $\Psi \ngtr \Phi_{n_{1}, n_{2}, \cdots, n_{k}}^{i}$ for every $i, k=1,2, \cdots$, the restriction of $\Psi$ in $E_{n_{1}, n_{2}, \cdots, n_{k}}$ is a Cauchy-filter 
for every $k=1,2, \cdots$, and hence, by the assumption, $\Psi$ converges in $E$. Thus $E$ is a $G N$-space.

Netted spaces of [1] and [2], and $\alpha \beta \gamma$-representable spaces of [3] are $G N$-spaces, because they are special cases of the linear topological space $E$ with a system of subspaces $E_{n_{1}, n_{2}, \ldots, n_{k}}$ satisfying the condition of the proposition, where the topology given on each $E_{n_{1}, n_{2}, \ldots, n_{k}}$ is metric.

Corresponding to the closed graph theorem for quasi-Souslin space in [4], we obtain the following

Theorem. Every graph closed linear mapping $\varphi$ from a linear topological space $F$ of second category into a $G N$-space $E$ is continuous.

Proof. Let $E$ be a $G N$-space with defining $P$-filters $\Phi_{n}$. If we can prove that there exists a sequence of subsets $A_{i}(i=1,2, \ldots)$ of $F$ such that $A_{i}$ is everywhere second category in $F, A_{i} \supset A_{i+1}$, and, for every $x$ in $A_{i}$, there exists a neighborhood $U$ of $x$ such that $U \cap A_{i}$ is disjoint from $\varphi^{-1}\left(\Phi_{i}\right)$, then the same argument as in the corresponding proof in [4] can be applied to complete our proof. We put $F=A_{0}$. Now we show that we obtain a sequence of subsets $A_{i}(i=1,2, \ldots)$ such that each $A_{i}$ satisfies the following condition (*) in addition to the above condition.

For every $x$ in $A_{i}$ there exist a linearly open set $L \ni x$ in $F$

(*) and a second category linear subspace $H \ni x$ of $F$ such that $A_{i} \supset L \cap H$.

Clearly $A_{0}$ satisfies the condition (*). For each $i$ when $A_{i}$ is already determined, we determine $A_{i+1}$ in the following way. Let $\left\{\left(V_{\lambda}, B_{\lambda}\right)\right\}_{\lambda \in A}$ be a maximal family of pairs $\left(V_{\lambda}, B_{\lambda}\right)$ with the following conditions (1) to (4):

(1) $B_{\lambda}$ is everywhere second category in non-void open set $V_{\lambda}$.

(2) $A_{i} \supset B_{\lambda}, B_{\lambda}$ is disjoint from $\varphi^{-1}\left(\Phi_{i+1}\right)$.

(3) $B_{\lambda}$ satisfies the condition $(*)$.

(4) $V_{\lambda} \cap V_{\lambda^{\prime}}=\emptyset$ if $\lambda \neq \lambda^{\prime}$.

Put $A_{i+1}=\bigcup_{\lambda \in \Lambda} B_{\lambda}$. Suppose there exists an open set $W$ such that $W \cap V_{\lambda}$ $=\emptyset$ for all $\lambda \in \Lambda$. For $x$ in $W \cap A_{i}$, there exist a linearly open set $L \ni x$ in $F$ and a second category linear subspace $H \ni x$ of $F$ such that $W \cap A_{i} \supset L \cap H \ni x$. As $\Phi_{i+1}$ is a $P$-filter in $E, \varphi^{-1}\left(\Phi_{i+1}\right)$ is also a $P$-filter in $F$. So there exists a linearly open set $M \ni x$ in $F$ such that $M$ is either disjoint from $\varphi^{-1}\left(\Phi_{i+1}\right)$ or $\left\{\varphi^{-1}\left(\Phi_{i+1}\right)\right\}_{M}$ is finer than a $L S$-filter $\Psi$ defined by a sequence of subspaces $F_{k}(k=1,2, \ldots)$. Put $C=L \cap H \cap M$ in the first case, and, in the second case, $C=L \cap H \cap M \cap F_{k}$ where $k$ is chosen as to let $C$ be second category. Putting $V=O(C)$ and $B=V \cap C$, we obtain a pair $(V, B)$ and the family $\left\{\left(V_{\lambda}, B_{\lambda}\right),(V, B)\right\}$ satisfying (1), (2), (3), and (4), contradicting the maximality of $\left\{\left(V_{\lambda}, B_{\lambda}\right)\right\}_{\lambda \in \Lambda}$. So we 
have proved that $\bigcup_{\lambda \in A} V_{\lambda}$ is dense in $F$, and then it is obvious that $A_{i+1}$ satisfies all the required conditions.

\section{References}

[1] H. G. Garnir: Some new results in classical functional analysis. Proceedings of the International Conference on Functional Analysis and Related Topics, 361-368 (1970).

[2] M. De Wilde: Sur le théorème du graphe fermé. C. R. Acad. Sc. Paris, 265, série A, 376-379 (1967).

[ 3 ] W. Slowikowski: On continuity of inverse operators. Bull. Amer. Math. Soc., 67 (5), 467-470 (1961).

[4] M. Nakamura: On quasi-Souslin space and closed graph theorem. Proc. Japan Acad., 46 (6), 514-517 (1970). 